\title{
De novo hepatocellular carcinoma occurrence in hepatitis $C$ cirrhotics treated with direct-acting antiviral agents
}

\author{
Gabriela Kuftinec', Thomas Loehfelm², Michael Corwin², Blythe Durbin-Johnson ${ }^{3}$, \\ MarieChristi Candido ${ }^{4}$, Rebecca Hluhanich**,5 \& Souvik Sarkar*,4 \\ ${ }^{1}$ Department of Internal Medicine,University of California Davis, 4150 V St, PSSB 3100, Sacramento, CA 95817, USA \\ ${ }^{2}$ Department of Radiology, University of California Davis, 4860 Y Street, Suite 3100, Sacramento, CA 95817, USA \\ ${ }^{3}$ Division of Biostatistics, University of California Davis, Genome and Biomedical Sciences Facility, 451 Health Sciences Dr., Rm. \\ 1300, Davis, CA 95616, USA \\ ${ }^{4}$ Division of Gastroenterology \& Hepatology, University of California Davis, 4150 V St, PSSB 3500, Sacramento, CA 95817, USA \\ ${ }^{5}$ Hepatology \& Infectious Diseases Specialty Pharmacy, University of California Davis Health, 2315 Stockton Blvd, Sacramento, CA \\ 95817, USA \\ *Author for correspondence: Tel.: +1 916734 3759; ssarkar@ucdavis.edu \\ **Author for correspondence: Tel.: +1 916734 0900; rhluhanich@ucdavis.edu
}

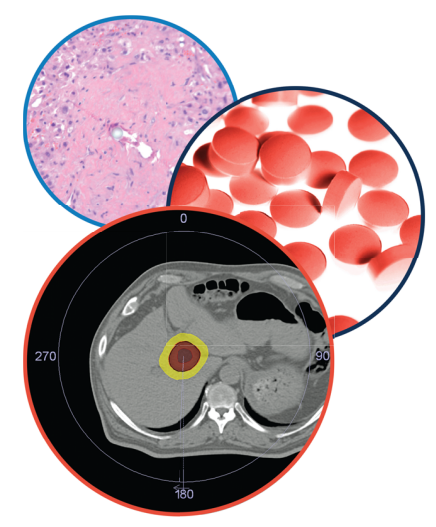

Hepatic Oncology

\begin{abstract}
Aim: Recent studies raise concerns for higher incidence of hepatocellular carcinoma (HCC) after directacting antiviral therapy for hepatitis $\mathrm{C}$ virus (HCV). Methods: In this study, using analysis of liver imaging pre- and post-DAA treatment, we queried new occurrence or 'de novo' of HCC in patients with HCVcirrhosis treated with DAAs. Of 150 patients who met study criteria, 7 (4.7\%; $95 \% \mathrm{Cl}: 2.1-9.5 \%)$ patients developed de novo HCC which did not differ from historical rates of $3 \%(p=0.22)$. Results: Notably, patients with decompensated cirrhosis had significantly higher rate of de novo HCC $(9.3 \%$; $95 \% \mathrm{Cl}$ : $3.12-$ $22.2 \% ; p=0.04)$. Conclusion: Our data support the need for continued surveillance for HCC in HCV cirrhotics even after successful therapy.
\end{abstract}

First draft submitted: 22 January 2018; Accepted for publication: 4 May 2018; Published online: 25 July 2018

Keywords: cirrhosis • de novo HCC • direct-acting antivirals • hepatitis C virus $\bullet$ hepatocellular carcinoma $\bullet$ interferon $\bullet$ LiRADS 4, $5 \bullet$ liver-directed imaging

Hepatitis C virus (HCV) infection affects nearly 3 million individuals in the USA [1] and up to 185 million affected patients worldwide [2,3]. Deaths due to HCV complications have risen since 2010 and will likely continue to increase over the next several years [4]. In patients infected with HCV, about $20 \%$ will eventually develop liver cirrhosis, and a substantial proportion of those will go on to develop hepatocellular carcinoma (HCC) [5]. HCC is the fifth most common type of cancer worldwide and is the second leading cause of cancer-related deaths [6].

The majority of HCC cases are found in patients with cirrhosis of the liver [7]. For patients chronically infected with $\mathrm{HCV}$, the progression to cirrhosis is promoted by HCV-induced inflammation. While HCV infection is the strongest risk factor for development of HCC, other risk factors include male sex, coinfection with hepatitis B virus or HIV, alcohol consumption and older age. Of HCC cases in the USA, 50-60\% are secondary to HCV. It is estimated that the annual incidence of HCC development after cirrhosis develops is about $1-4 \%$, with a time-period of roughly 20 years from initial HCV infection to development of HCC without any treatment [7].

During the past several years, major innovations have been made in the HCV treatment realm, with new directacting antiviral (DAA) regimens achieving greater than a $90 \%$ sustained virologic response (SVR) rate for most viral genotypes. Studies have shown rates of SVR around $97 \%$ in compensated cirrhotics [8-10] and up to $90 \%$ in patients with more advanced liver disease, some even awaiting transplant [11,12]. These therapies are taken orally and are interferon-free, virtually eliminating the significant side effects of interferon therapy that once represented a large barrier to successful treatment. The increased effectiveness and tolerability of the new class of drugs allows for treatment of patients with much more advanced disease [13].

Future Medicine 
Previous interferon-based regimens have been shown to possibly decrease the risk of developing new HCC and to decrease rate of recurrence [14,15]. Currently, data are conflicting on the impact of DAAs on the development of HCC. Several studies showed that patients infected with HCV who have received treatment with DAA remain at risk for HCC and with potential increased risk for recurrence, ranging from 6.6 to $9.1 \%$ [16-20]. Conversely, other studies showed reductions in HCC occurrence after achievement of SVR from DAA-containing therapies [21-24]. In a large study of over 60,000 patients receiving combinations of interferon only, DAA+interferon and DAA only, SVR was again associated with a significantly decreased risk of HCC irrespective of the type of treatment. DAA treatment was found to be associated with $71 \%$ risk reduction of development of HCC [22].

A systematic review and meta-analysis also found that DAA therapy was not associated with higher HCC occurrence. Higher incidence of HCC was observed in older patients and with shorter follow-up periods [24]. In view of these conflicting reports, we specifically investigated the new occurrence of HCC in patients with HCV cirrhosis who underwent treatment with DAAs, utilizing radiologic evaluation for lesions pre- and post-treatment.

\section{Methods}

\section{Patient population}

This retrospective, single-center study investigated the development of de novo HCC in patients with HCV cirrhosis who received treatment with a DAA-containing regimen at University of California, Davis Health (CA, USA) from 1 January, 2014 to 1 April, 2017. Institutional Review Board approval was obtained. Patients were included in the study if they met the following criteria: age $\geq 18$ years; had chronic hepatitis $\mathrm{C}$; had cirrhosis as defined by either clinical status, labs, imaging, FIB-4 score or a combination of these; treated with DAA-containing regimen; had liver-directed imaging (screening ultrasound, CT scan or MRI) up to 12 months prior to treatment; had liver-directed imaging 12 months postcompletion of DAA treatment and if any lesion was detected, patient needed to have 4-phase CT scan or dynamic MRI to be in the final cohort. Patients were excluded if they met the following criteria: minors; fibrosis/METAVIR Stage 0-3; patients without both pre- and post-imaging within 12 months of treatment or without dynamic imaging, if any lesion was detected; incarcerated patients; pregnant patients; prior history of liver transplant and presence of LI-RADS 4 4 lesions prior to receiving DAA therapy.

\section{Study objectives}

The primary objective of the study was to determine new occurrence of HCC as defined as LIRADS 4 or 5 after completion of HCV treatment with DAA-based regimens. Imaging was either screening ultrasound, CT scan or MRI. If there was a lesion that was identified on those imaging studies, a multiphase CT or MRI was then required to define HCC. Two independent reviewers performed a retrospective chart review of these patients to determine if there was 'no', 'new' or 'pre-existent' HCC. The secondary objectives of the study evaluated linkage to genotype, SVR or regimen for the occurrence of DAAs. HCC was defined by radiologic criteria on multiphase imaging either as definite HCC or LIRADS $4 / 5$ on the radiology report. A radiologist would review any conflicts on LIRADs classification.

\section{Hepatitis $\mathrm{C}$ antiviral treatment}

All patients underwent HCV treatment with a DAA-based regimen for either 12 or 24 weeks as indicated by the American Association for the Society of Liver Diseases/Infectious Diseases and Society of America HCV treatment guidelines. Treatment regimen selection was chosen at that discretion of the physician. Baseline characteristics, laboratory values, radiologic tumor response were recorded prior to initiation of antiviral therapy.

\section{Time-points for radiologic evaluation of tumor}

Radiological studies, either abdominal ultrasound, 4-phase CT scan or MRI were evaluated for each study participant at two time points. The first evaluation for HCC was within the 12-month period prior to initiation of DAA treatment. All dynamic images between 12 months prior to the start of treatment were reviewed to evaluate for any pre-existing lesion. The second time-point was within 12 months after the end of treatment.

\section{Statistical analysis}

The data were analyzed by a statistician as a noninferiority study. The exact binomial test was used to test for statistical significance. Confidence intervals for proportions were calculated using the Agresti-Coull method [25]. 


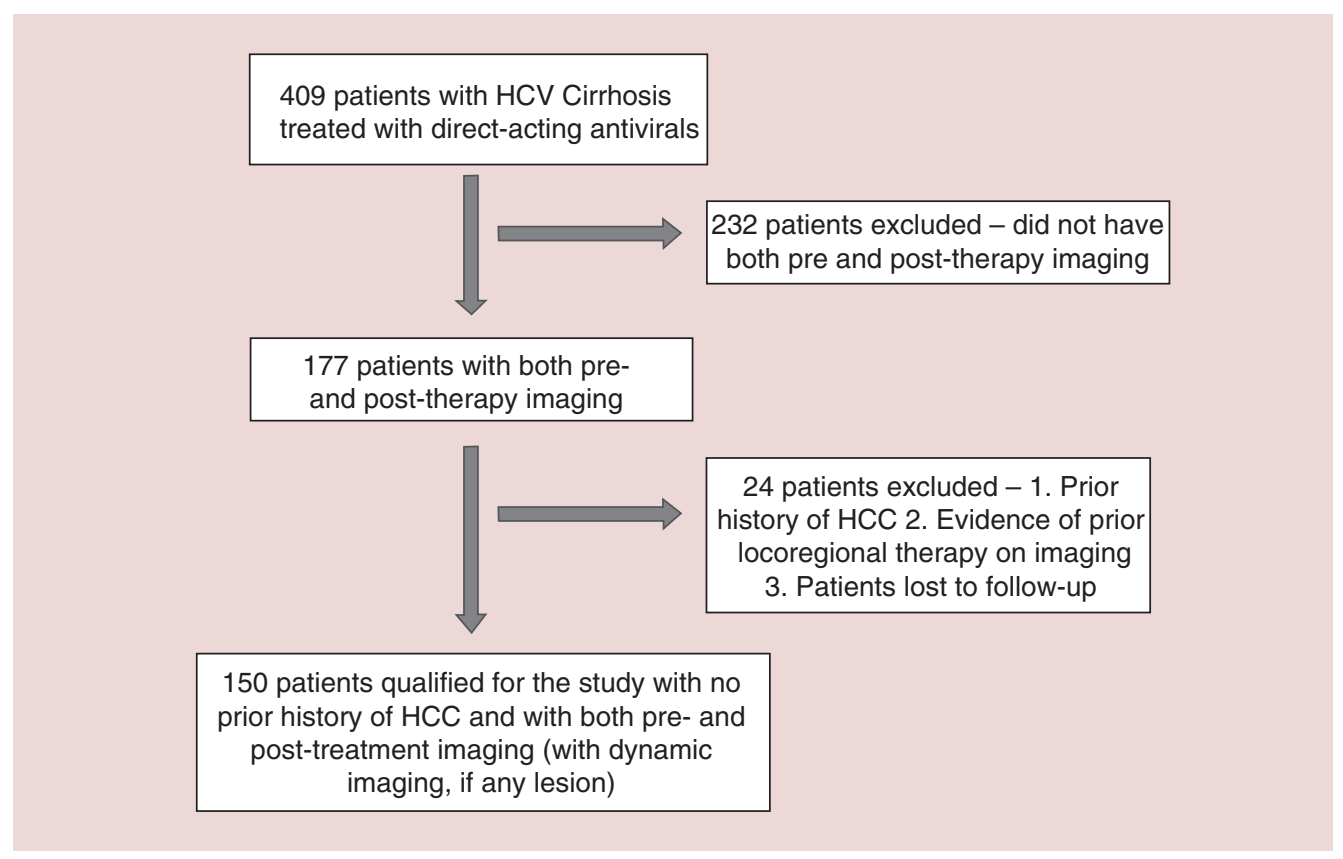

Figure 1. Study enrollment. This flowchart depicts the study inclusion and exclusion criteria utilized to select the patient population.

Proportions were compared with a historical proportion of $3 \%$ using an exact binomial test. Analyses were conducted using the statistical software environment $\mathrm{R}$, version 3.4.1 [26] and the R package binom, version 1.1-1 [27].

\section{Results}

\section{Demographics}

From 1 January 2014 to 1 April 2017, 409 patients with cirrhosis were treated with a DAA-containing regimen at the University of California, Davis Health. Of these patients, 150 met the inclusion criteria as shown in Figure 1. A total of 146 patients were treated with an all-oral DAA regimen, whereas four patients were treated with sofosbuvir, interferon and ribavirin. The majority of the patients included in the study were males (59\%), with compensated cirrhosis (71\%) and infected with HCV genotype 1 (77\%). Half of the patients were treated with a sofosbuvir/ledipasvir-containing regimen and the most patients achieved SVR (93\%) (Table 1).

\section{New HCC occurrence after DAA completion}

Seven of 150 patients (4.67\%; CI: 2.1-9.5) developed de novo HCC within 12 months from completion of HCV treatment. Of the seven patients, six were noted to have LIRADS 5 and one with LIRADS 4 lesion on multiphase imaging. These patients had pretreatment imaging 12 months prior to starting treatment with DAAs that did not show any evidence of HCC. When compared with historical rates of 3\% for new HCC occurrence in $\mathrm{HCV}$ cirrhosis, the observed rate in this study was not considered statistically different ( $\mathrm{p}$-value $=0.224)$. For patients treated with an all-oral DAA regimen, the rate of de novo HCC development slightly increased to $4.79 \%$ ( $\mathrm{n}=7 / 146$, CI (2.16-9.74\%) and was also not statistically different from the historical rate (p-value $=0.215)$.

New lesions were mostly identified within 0-6 months post-treatment ( $\mathrm{n}=5 / 7 ; 71.4 \%$ ). No new lesions were identified during the HCV treatment. The majority of patients were HCV treatment naive prior to the DAA-containing therapy $(n=5 / 7 ; 71.4 \%)$ and $57 \%$ of patients $(n=4 / 7)$ had decompensated cirrhosis. Patients developing de novo HCV were either infected with HCV genotype 1A, 3 or 6. All patients achieved SVR12 (Table 2).

Rates of de novo HCC were significantly higher in patients with decompensated cirrhosis at $9.3 \%$ when compared with historical rates of $3 \%$ ( $\mathrm{p}$-value $=0.040$ ) and were threefold higher than rates observed compensated cirrhosis, $9.3 \%$ compared with $2.8 \%$, respectively. While rates of de novo HCC were observed to be higher in patients who 
Table 1. Patient demographics.

\begin{tabular}{|c|c|c|c|}
\hline Primary characteristics & Sub-characteristics & n & Total percentage \\
\hline Overall & - & 150 & 100 \\
\hline \multirow[t]{2}{*}{ Viral response } & SVR & 140 & 93 \\
\hline & Relapse & 10 & 7 \\
\hline \multirow[t]{2}{*}{ Gender } & Male & 89 & 59 \\
\hline & Female & 61 & 41 \\
\hline \multirow[t]{3}{*}{ Race/ethnicity } & Asian & 3 & 2 \\
\hline & White & 46 & 31 \\
\hline & Unknown & 89 & 59 \\
\hline \multirow[t]{3}{*}{ BMI } & $<25$ & 43 & 29 \\
\hline & $25-29.9$ & 50 & 33 \\
\hline & $30-35$ & 33 & 22 \\
\hline \multirow{4}{*}{ HCV genotype } & 2 & 11 & 7 \\
\hline & 3 & 19 & 13 \\
\hline & 4 & 1 & 1 \\
\hline & 6 & 4 & 3 \\
\hline \multirow[t]{2}{*}{ Compensation } & Compensated & 107 & 71 \\
\hline & Decompensated & 43 & 29 \\
\hline \multirow[t]{4}{*}{ Treatment regimen } & $\mathrm{SOF}+\mathrm{LDV}+/-\mathrm{RBV}$ & 74 & 49 \\
\hline & $\mathrm{SIM}+\mathrm{SOF}+/-\mathrm{RBV}$ & 33 & 22 \\
\hline & $\mathrm{SOF}+\mathrm{RBV}$ & 20 & 13 \\
\hline & GRZ+ELB & 6 & 4 \\
\hline
\end{tabular}

were treatment-naive prior to receiving the DAA-containing therapy or infected with HCV genotype 1A, 3 or 6 , the differences were not statistically significant compared with historical rates (Table 3).

\section{Discussion}

HCC is a potentially fatal complication of HCV-related cirrhosis with an annual incidence rate around 3.5\% [20]. Prior to the advent of DAA-therapies, HCV treatment with PEG-IFN and RBV was associated with a decreased risk for HCC development. De novo HCC rates in patients treated with IFN who achieve SVR have been reported to be $0.5-2 \%$ over 3 years post-treatment [15,28-31]. This benefit was anticipated to translate to the direct-acting agents; however, several studies suggest that there may be an increase in rates of de novo and recurrent HCC after HCV treatment with DAAs. Reported rates of de novo HCC status post DAA-based therapy have ranged from 6.6\% to $9.1 \%$ [16-20]. However, these studies did have limitations, as they were mostly small, retrospective, uncontrolled studies with short-term follow-up. Additionally, their sample population was drawn from patients at higher risk of HCC, such as over age 60 or already with liver failure. More recent studies suggest either no change in the rate of HCC after successful HCV treatment with DAA therapies or a similar decrease compared with IFN-based regimens [21-24]. In two larger retrospective studies, the risk of HCC was reduced by $71-76 \%$ in patients who achieved SVR being treated with DAA-containing regimen [21,22]. In the study conducted by the Veterans Health Administration, patients with cirrhosis were found to have the highest risk of HCC [21]. Moreover, a systematic 
Table 2. Characteristics of patients with de novo hepatocellular carcinoma occurrence.

\begin{tabular}{|c|c|c|c|}
\hline Primary characteristics & Sub-characteristics & $\mathrm{n}$ & $\%$ total \\
\hline Overall new HCC & & 7 & $4.7 \%$ \\
\hline Age & $>40-70$ & 7 & $100 \%$ \\
\hline \multirow[t]{2}{*}{ Gender } & Male & 4 & $57.1 \%$ \\
\hline & Female & 3 & $42.9 \%$ \\
\hline \multirow[t]{2}{*}{ Cirrhosis } & Compensated & 3 & $43.0 \%$ \\
\hline & Decompensated & 4 & $57.0 \%$ \\
\hline \multicolumn{4}{|l|}{ Laboratory values at baseline } \\
\hline ALT & $>40$ & 3 & $43 \%$ \\
\hline \multirow[t]{2}{*}{ Platelet } & $\leq 150$ & 6 & $86 \%$ \\
\hline & $>150$ & 1 & $14 \%$ \\
\hline \multirow[t]{2}{*}{ Time to diagnosis of new HCC } & $0-6$ months post-treatment & 5 & $71.4 \%$ \\
\hline & $7-12$ months post-treatment & 2 & $28.6 \%$ \\
\hline \multirow{2}{*}{ HCV genotype } & 3 & 2 & $1.3 \%$ \\
\hline & 6 & 1 & $0.7 \%$ \\
\hline \multirow[t]{2}{*}{ Treatment status } & Treatment-naive & 5 & $71.4 \%$ \\
\hline & $\begin{array}{l}\text { Treatment-experienced (IFN or } \\
\text { IFN/DAA) }\end{array}$ & 2 & $28.6 \%$ \\
\hline Treatment response & SVR & 7 & $100 \%$ \\
\hline
\end{tabular}

ALT: Alanine aminotransferase; DAA: Direct-acting anitiviral; HCC: Hepatocellular carcinoma; HCV: Hepatitus C virus; IFN: Interferon; LIRADS: Liver reporting and data system; SVR: Sustained virologic response.

Table 3. Rates of de novo hepatocellular carcinoma per patient populations.

\begin{tabular}{|c|c|c|c|c|}
\hline Group & Number of new HCC & Total number of patients & Rate $(95 \% \mathrm{Cl})$ & p-value ${ }^{\dagger}$ \\
\hline Treatment-naive & 5 & 87 & $5.75 \%(2.16 \%, 13.07 \%)$ & 0.192 \\
\hline Treatment-experienced & 2 & 63 & $3.17 \%(0.23 \%, 11.50 \%)$ & 0.714 \\
\hline Compensated & 3 & 107 & $2.80 \%(0.61 \%, 8.27 \%)$ & $>0.999$ \\
\hline Decompensated & 4 & 43 & $9.30 \%(3.12 \%, 22.16 \%)$ & 0.040 \\
\hline Male & 4 & 89 & $4.49 \%(1.41 \%, 11.35 \%)$ & 0.345 \\
\hline Female & 3 & 61 & $4.92 \%(1.14 \%, 14.03 \%)$ & 0.433 \\
\hline GT 1a & 4 & 77 & $5.19 \%(1.64 \%, 13.00 \%)$ & 0.296 \\
\hline GT 3 & 2 & 19 & $10.53 \%(1.70 \%, 32.63 \%)$ & 0.110 \\
\hline GT 6 & 1 & 4 & $25.00 \%(3.41 \%, 71.09 \%)$ & 0.115 \\
\hline
\end{tabular}

$\dagger p$-value of $<0.05$ is considered statistically significant from historical HCC rate of $3 \%$ (Bold font indicates that rates of de novo HCC were significantly higher in patients with decompensated cirrhosis)

GT: Genotype; HCC: Hepatocellular carcinoma.

review and meta-analysis found that DAA therapy was not associated with higher HCC occurrence. Incidence of $\mathrm{HCC}$ was lower in younger patients and in those patients who were followed for a longer period of time [24].

This study investigated the incidence of de novo HCC in cirrhotic patients, both compensated and decompensated, who were treated with DAA-containing therapy and followed with radiologic imaging within 12 months after completion of HCV treatment. Overall, no significant difference was observed in rates of de novo HCC in patients treated with DAA-containing therapy when compared with historical rates. While data are conflicting regarding the impact of DAA-therapy on the rate of HCC, this study provides evidence that DAAs did not significantly increase the risk for HCC. The caveat remains that the risk is not appreciably decreased compared with historical rates or as noted with patients treated with interferon-based therapies.

As DAA therapy has a substantially improved safety profile compared with interferon, more complicated patients are being treated for $\mathrm{HCV}$, including those with decompensated cirrhosis. Interferon use is contraindicated in 
Child's Turcotte Pugh Class B and C patients; thus, excluding these patients from the earlier studies evaluating the rates of HCC development status post-treatment. In this study, patients with decompensated cirrhosis were found to have a significantly higher rate of de novo HCC development at $9.3 \%$ compared with historical rates. Nearly $60 \%$ of the patients who developed de novo HCC had decompensated cirrhosis and the rate of de novo HCC development in decompensated cirrhotic patients was threefold higher than in compensated cirrhotic patients, $9.3 \%$ compared with $2.8 \%$, respectively.

Decompensated cirrhosis has been shown to be an independent risk factor for developing HCC and is possibly contributing to the higher rates of HCC observed in cirrhotic patients treated with DAA-containing regimens [32,33]. Similar to the data presented in this study, a surveillance program conducted in 313 Italian patients with cirrhosis of differing etiologies identified Child Pugh Class B/C as an independent risk factor the development of HCC, with patients having a threefold increased risk [32]. In a prospective study in 400 Chinese patients with either hepatitis B or HCV cirrhosis, Child Pugh Class B was also associated with a threefold increased risk while Child Pugh Class $\mathrm{C}$ was associated with a eightfold higher risk of developing HCC [33].

Time to new HCC lesion identification occurred after completion of DAA-containing therapy for all seven patients developing de novo HCC in this study. For the majority of these patients, identification occurred within 0-6 months after HCV treatment; thus, highlighting the importance of continued HCC monitoring every 6 months for HCV cirrhosis irrespective of the HCV treatment.

One of the key strengths of the study is rigorous definition of de novo HCC. Cirrhotic patients with 'no prior lesion' by definition is only in patients without any prior HCC, either treated or untreated. Such a cohort will be difficult to ascertain with electronic search criteria. Thus, imaging was reviewed of all pre- and post-treated patients to evaluate for any lesions. Any patients with positive lesions needed to have a dynamic imaging to rule out HCC. All patients with a post-treatment lesion were evaluated with two independent reviewers for any past history of HCC by chart review and/or history of any HCC treatment noted by an ablation cavity on imaging. This process helped in eliminating patients who would otherwise be considered a 'recurrence' with risk of altering the true overall occurrence rate. The major limitation to this study is that it is a small, single-center retrospective cohort with an uncontrolled design and brief follow-up period. Therefore, it is difficult to fully appreciate the entire impact of DAA treatment on HCC risk. Larger studies with longer follow-up durations are needed to further evaluate this long-term benefits and possible risk for DAA-containing therapies and their possible impact on HCC development. Additionally, because our cohort was made up of entirely cirrhotic patients with higher baseline risk of HCC development than their noncirrhotic counterparts, it is a possibility that our baseline risk may be skewed above historical rates, that took into account cirrhotic and noncirrhotic patients. Further, our cohort of patients with liver failure was quite heterogeneous, with $43 / 150$ patients in decompensated cirrhosis. This could have led any potential positive effects of DAA treatments being missed; however, given the lack of data from studies such as ours, we argue that the historical rates used for comparison are still reasonable. We did not specifically investigate whether other comorbidities such as nonalcoholic fatty liver disease or nonalcoholic steatohepatitis affected the HCC occurrence but would be an excellent area of future research.

\section{Conclusion}

In conclusion, this study showed no increase in the rate of de novo HCC in HCV cirrhotic patients treated with DAA-containing therapy during a 12-month monitoring window compared with historic rates; however, patients with decompensated cirrhosis were found to have a significantly higher rate of new HCC occurrence. As decompensated cirrhotic patients were excluded from earlier interferon studies, rates of HCC may be increased in the DAA-treatment era given the new ability to treat patients with more advanced liver disease. This data does support the continued need for routine HCC monitoring in HCV cirrhotic patients after receiving antiviral therapy.

\section{Future perspective}

We believe that this topic will continue to be of interest over the next several years, with ongoing study and data collection. As we collect long-term follow-up data on patients with HCV cirrhosis treated with DAAs, the risk for HCC will be more apprehensible. Until we fully understand the risks for HCC, patients with HCV cirrhosis should continue to be screened according to current national guidelines even after achieving viral eradication. 
Summary points

- Several conflicting studies have recently been published investigating rates of hepatocellular carcinoma (HCC) occurrence after treatment of hepatitis $\mathrm{C}$ virus (HCV) with new oral direct acting antivirals (DAA) agents.

- While treatment of patients with HCV cirrhosis with novel DAAs have yielded good results in terms of achieving sustained virologic response, the development of HCC in these patients is still unknown.

- To help address this question, this study investigated the development of de novo HCC in HCV cirrhotic patients treated with DAAs in a small, single-center retrospective cohort study design.

- Patients with known HCV cirrhosis and liver-directed imaging at least 12 months prior to starting DAA treatment, as well as up to 12 months after completion of DAA treatment were included in the study.

- Any patients with lesions prior to initiation of DAA treatment were excluded.

- Imaging studies were reviewed by two independent reviewers for development of a LiRADS 4 or 5 lesion.

- $4.6 \%$ of the patients developed HCC within 0-6 months after completion of DAA therapy.

- Overall, no significant difference was observed in rates of de novo HCC in patients treated with DAA-containing therapy when compared with historical rates.

Financial \& competing interests disclosure

S Sarkar has served on advisory boards for Gilead sciences and Abbvie and received grants (paid to UC Davis) from Gilead Sciences. $\mathrm{R}$ Hluhanich has stocks in Gilead Sciences. The authors have no other relevant affiliations or financial involvement with any organization or entity with a financial interest in or financial conflict with the subject matter or materials discussed in the manuscript apart from those disclosed.

No writing assistance was utilized in the production of this manuscript.

\section{Ethical conduct of research}

The authors state that they have obtained appropriate institutional review board approval or have followed the principles outlined in the Declaration of Helsinki for all human or animal experimental investigations. In addition, for investigations involving human subjects, informed consent has been obtained from the participants involved.

(Note: The study was approved by the UC Davis Institutional Review Board and exempted from obtaining consents)

\section{Open access}

This work is licensed under theAttribution-NonCommercial-NoDerivatives 4.0 Unported License. To view a copy of this license, visit http://creativecommons.org/licenses/by-nc-nd/4.0/

\section{References}

Papers of special note have been highlighted as: $\bullet$ of interest; $\bullet \bullet$ of considerable interest

1. Klevens RM, Hu DJ, Jiles R, Holmberg SD. Evolving epidemiology of hepatitis C virus in the United States. Clin. Infect. Dis. 55(Suppl. 1) S3-S9 (2012).

2. Denniston MM, Jiles RB, Drobeniuc J et al. Chronic hepatitis C virus infection in the United States, National Health and Nutrition Examination Survey 2003-2010. Ann. Intern. Med. 160(5), 293-300 (2014).

3. Mohd Hanafiah K, Groeger J, Flaxman AD, Wiersma ST. Global epidemiology of hepatitis C virus infection: new estimates of age-specific antibody to HCV seroprevalence. Hepatology 57(4), 1333-1342 (2013).

4. Zoulim F, Liang TJ, Gerbes AL et al. Hepatitis C virus treatment in the real world: optimizing treatment and access to therapies. Gut 64(11), 1824-1833 (2015).

5. Kohli A, Shaffer A, Sherman A, Kottilil S. Treatment of hepatitis C: a systematic review. JAMA 312(6), 631-640 (2014).

6. Ferlay J, Soerjomataram I, Dikshit R et al. Cancer incidence and mortality worldwide: sources, methods and major patterns in GLOBOCAN 2012. Int. J. Cancer 136(5), E359-E386 (2015).

7. El-Serag HB. Epidemiology of viral hepatitis and hepatocellular carcinoma. Gastroenterology 142(6), 1264-1273. e1261 (2012).

8. Lawitz E, Makara M, Akarca US et al. Efficacy and safety of ombitasvir, paritaprevir and ritonavir in an open-label study of patients with genotype $1 \mathrm{~b}$ chronic hepatitis C virus infection with and without cirrhosis. Gastroenterology 149(4), 971-980 (2015).

9. Poordad F, Hezode C, Trinh R et al. ABT-450/r-ombitasvir and dasabuvir with ribavirin for hepatitis $\mathrm{C}$ with cirrhosis. N. Engl. J. Med. 370(21), 1973-1982 (2014).

10. Reddy KR, Bourliere M, Sulkowski $M$ et al. Ledipasvir and sofosbuvir in patients with genotype 1 hepatitis $\mathrm{C}$ virus infection and compensated cirrhosis: an integrated safety and efficacy analysis. Hepatology 62(1), 79-86 (2015). 
11. Charlton M, Everson GT, Flamm SL et al. Ledipasvir and sofosbuvir plus ribavirin for treatment of HCV infection in patients with advanced liver disease. Gastroenterology 149(3), 649-659 (2015).

12. Poordad F, Schiff ER, Vierling JM et al. Daclatasvir with sofosbuvir and ribavirin for hepatitis $\mathrm{C}$ virus infection with advanced cirrhosis or post liver transplantation recurrence. Hepatology 63(5), 1493-1505 (2016).

13. Webster DP, Klenerman P, Dusheiko GM. Hepatitis C. Lancet 385(9973), 1124-1135 (2015).

14. Ikeda K, Saitoh S, Arase Y et al. Effect of interferon therapy on hepatocellular carcinogenesis in patients with chronic hepatitis type C: a long-term observation study of 1643 patients using statistical bias correction with proportional hazard analysis. Hepatology 29(4), 1124-1130 (1999).

-. Evaluates effect on interferon therapy and hepatocellular carcinoma (HCC).

15. Hiramatsu N, Oze T, Takehara T. Suppression of hepatocellular carcinoma development in hepatitis C patients given interferon-based antiviral therapy. Hepatol. Res. 45(2), 152-161 (2015).

16. Reig M, Marino Z, Perello C et al. Unexpected high rate of early tumor recurrence in patients with HCV-related HCC undergoing interferon-free therapy. J. Hepatol. 65(4), 719-726 (2016).

-• Landmark paper ignites interest with regards to risk of HCC recurrence after direct-acting antiviral therapy for hepatitis $\mathrm{C}$ virus.

17. Cardoso H, Vale AM, Rodrigues $S$ et al. High incidence of hepatocellular carcinoma following successful interferon-free antiviral therapy for hepatitis C associated cirrhosis. J. Hepatol. 65(5), 1070-1071 (2016).

- Follows the above article.

18. Ravi S, Axley P, Jones D et al. Unusually high rates of hepatocellular carcinoma after treatment with direct-acting antiviral therapy for hepatitis C related cirrhosis. Gastroenterology 152(4), 911-912 (2017).

19. Toyoda H, Kumada T, Tada T. Changes in patient backgrounds may increase the incidence of HCC after SVR in the era of IFN-free therapy for HCV. Hepatology 64(5), 1818-1819 (2016).

20. Conti F, Buonfiglioli F, Scuteri A et al. Early occurrence and recurrence of hepatocellular carcinoma in HCV-related cirrhosis treated with direct-acting antivirals. J. Hepatol. 65(4), 727-733 (2016).

21. Kanwal F, Kramer J, Asch SM, Chayanupatkul M, Cao Y, El-Serag HB. Risk of hepatocellular cancer in HCV patients treated with direct-acting antiviral agents. Gastroenterology 153(4), 996-1005, e1001 (2017).

-• Shows decrease in overall HCC occurrence after sustained virologic response but no change in HCC incidence in cirrhotic patients.

22. Ioannou GN, Green PK, Berry K. HCV eradication induced by direct-acting antiviral agents reduces the risk of hepatocellular carcinoma. J. Hepatol. doi:10.1016/j.jhep.2017.08.030 (2017). (Epub ahead of print).

- Shows reduction of risk for HCC after sustained virologic response.

23. Nagata $\mathrm{H}$, Nakagawa $\mathrm{M}$, Asahina $\mathrm{Y}$ et al. Effect of interferon-based and -free therapy on early occurrence and recurrence of hepatocellular carcinoma in chronic hepatitis C. J. Hepatol. 67(5), 933-939 (2017).

24. Waziry R, Hajarizadeh B, Grebely J et al. Hepatocellular carcinoma risk following direct-acting antiviral HCV therapy: a systematic review, meta-analyses and meta-regression. J. Hepatol. 67(6), 1204-1212 (2017).

- Meta-analysis for HCC after direct-acting antiviral therapy for hepatitis $\mathrm{C}$ virus.

25. Agresti A, Coull BA. Approximate is better than 'exact' for interval estimation of binomial proportions. The American Statistician 52(2), 119-126 (1998).

26. R: a language and environment for statistical computing. (2017). www.R-project.org/

27. Dorai-Raj S. Binom: binomial confidence intervals for several parameterizations. (2014). https://CRAN.R-project.org/package=binom

28. van der Meer AJ, Veldt BJ, Feld JJ et al. Association between sustained virological response and all-cause mortality among patients with chronic hepatitis C and advanced hepatic fibrosis. JAMA 308(24), 2584-2593 (2012).

29. Morgan RL, Baack B, Smith BD, Yartel A, Pitasi M, Falck-Ytter Y. Eradication of hepatitis C virus infection and the development of hepatocellular carcinoma: a meta-analysis of observational studies. Ann. Intern. Med. 158(5 Pt 1), 329-337 (2013).

30. El-Serag HB, Kanwal F, Richardson P, Kramer J. Risk of hepatocellular carcinoma after sustained virological response in veterans with hepatitis C virus infection. Hepatology 64(1), 130-137 (2016).

31. Toyoda H, Kumada T, Tada $\mathrm{T}$ et al. Risk factors of hepatocellular carcinoma development in noncirrhotic patients with sustained virologic response for chronic hepatitis C virus infection. J. Gastroenterol. Hepatol. 30(7), 1183-1189 (2015).

32. Bolondi L, Sofia S, Siringo S et al. Surveillance programme of cirrhotic patients for early diagnosis and treatment of hepatocellular carcinoma: a cost-effectiveness analysis. Gut 48(2), 251-259 (2001).

33. Tsai JF, Jeng JE, Ho MS et al. Effect of hepatitis C and B virus infection on risk of hepatocellular carcinoma: a prospective study. $B r$. J. Cancer 76(7), 968-974 (1997). 